\title{
Decentralized Power Allocation for Cooperative Cognitive Radio Networks Based on Game Theory
}

\author{
Yang $\mathrm{Yu}$ \\ Wuhan No.2 Ship Development Design Institute, Wuhan, China \\ 544738692@qq.com
}

\begin{abstract}
Keywords: Cooperative communication, cognitive radio networks, power allocation, cooperative game, Nash bargaining solution
\end{abstract}

Abstract. Current studies demonstrate that cooperative communication between secondary users (SUs) can improve spectrum diversity in cognitive radio (CR) networks. However, any selfish SU in decentralized networks will not help other SU relay information with its otherwise wasted spectrum holes for nothing because the extra energy consumption will limit its own performance. In this paper, we investigate decentralized power allocation for cooperative CR networks. In our system, SU transmitters have the ability to transmit their own data and relay information for each other. Both SU transmit-receive (T-R) pairs aim at achieving optimal performance by consuming the same amount of energy under the transmit power and the primary user (PU) interference constraints. This power allocation problem can be formulated as a bargaining game, and the Nash bargaining solution (NBS) method is utilized to solve this problem. Numerical simulations and comparisons are presented to illustrate that the NBS power allocation achieve better fairness compared with the maximal sum rate scheme. And the NBS power allocation is efficient in that both SU T-R pairs perform better than if they work independently.

\section{Introduction}

As wireless communications continue to grow, the spectrum resources become more and more limited and valuable. However, a lot of licensed spectrum bands are still underutilized most of time. Cognitive radio (CR) [1] was proposed as the means to promote the efficient utilization of the spectrum by identifying unused licensed spectrum bands (spectrum holes) and exploiting them. Studies [2]-[3] have shown that spectrum holes may vary with different secondary users (SUs). Moreover, the traffic demands of SUs also can be quite different. Therefore, how to handle the unbalanced spectrum usage in cognitive radio networks (CRNs) to fulfill the heterogeneous traffic demands from SUs is an important problem.

The application of the cooperative technique can solve this problem to a great extent. By utilizing a SU which has rich spectrum holes as helper, to relay other SU's traffic with their otherwise wasted spectrum, the latter SU's performance can be significantly improved and communication can be established between a pair of SUs even if they have no common available bands [2]. Based on the research of [2], cooperative technique used for spectrum sharing has been studied in [3]-[6]. Among them, [3] developed power and channel allocation approaches for cooperative relay in CRNs. With the aim of achieving maximum throughput of the source, [6] studied the methods on relay selection and power allocation. In [4], a cognitive space-time-frequency coding technique was proposed. However, all these studies are based on the assumption that all SUs cooperate in centralized networks, which is not reasonable for selfish SUs in distributed CRNs. This is because relaying on abundant spectrum holes represents a cost of energy. A selfish SU only wants to consume its energy to optimize its own performance and will not act as a relay for nothing. Hence, in order to guarantee spontaneously cooperation in distributed CRNs, how to allocate power fairly and efficiently is an important problem.

Game theory is natural and powerful tool to tackle this kind of problem. In this area, [7]-[9] studied the problem of how a relay should allocate its power or bandwidth resource among multiple competing users, and [10] studied how to gain the highest profit for a user by selling its resource to other user. 
However, the research results were based on price mechanism and models where user acts as either a source or a potential relay. Moreover, CR scenario is not considered.

Compared with the studies in [7]-[10], we consider a symmetric model where two SU transmitter can transmit their own data and relay information for each other simultaneously, so each SU transmitter can trade its power resource for each other's relaying without price mechanism. We also consider the potential interference with primary users (PUs), which is very important to CRNs. Since both SU transmit-receive (T-R) pairs aim at achieving optimal performance by consuming the same amount of energy under the transmit power and the PU interference constraints. We can formulate this Power allocation problem as a bargaining game, and a unique solution of this game can be found by Nash bargaining solution (NBS).

The remainder of this paper is organized as follows. In Section II, we describe the symmetric cooperative system model. In Section III, we formulate the power allocation problem as a two-person bargaining game and find the NBS of the game. In Section IV, we present simulation results. Finally, we conclude this paper in Section V.

\section{System Model Description}

A four-node cooperative cognitive radio system model is illustrated in Fig.1. SU transmitters $t 1$ and $t 2$ regard SU receivers $r 1$ and $r 2$ as their destination respectively. We refer to a node pair including a source $s i(i=1,2)$ and its destination $d i$ as a SU pair $i$. The CR system coexists with a primary system and each SU node has sensed its available spectrum holes before data transmission. For the sake of simplicity, we assume that $t i$, $r i$ and $t j(j=2$ if $i=1$, and $j=1$ if $i=2)$ have common channel $\mathrm{CH} i$, so there is not only direct source-destination link but also source-relay-destination link in our system model. Node $s j$ can help relay information for SU pair $i$ and vice versa with discontiguous OFDM (D-OFDM) [2] which is adopted as the physical-layer technique at SU nodes $s 1$ and $s 2$. Without loss of generality, we assume that each channel has unit channel bandwidth to facilitate our discussion. We also assume the channel gains are known at all the SU nodes and constant during a time slot. Suppose that the amplify-and-forward (AF) relaying protocol is employed in this paper. An AF cooperative transmission procedure of SU pair $i$ occurs in one frame. Each transmission frame has a length of $T$ seconds and consists of two time slots. Because of the symmetry of the two SU pairs, we will only analyze SU pair 1 's performance.

In the first time slot, SU source node $s 1$ broadcasts its own information to both node $d 1$ and $s 2$ over $\mathrm{CH} 1$. The received signal $Y_{s 1}^{d 1}$ at node $d 1$ is given by

$$
Y_{s 1}^{d 1}[1]=\sqrt{p_{s 1}[1] g_{s 1}^{d 1}[1]} X_{s 1}[1]+Z_{s 1}^{d 1}[1],
$$

where $p_{s 1}[1]$ is the transmission power of $s 1, g_{s 1}^{d 1}[1]$ is the channel gain from node $s 1$ to node $d 1$, $X_{s 1}[1]$ is the transmitted data symbol of $s 1$, and $Z_{s 1}^{d 1}[1]$ is the additive white Gaussian noise (AWGN) in the channel between $s 1$ and $d 1$ in the first time slot. And the received signal $Y_{s 1}^{s 2}$ at node $s 2$ is

$$
Y_{s 1}^{s 2}[1]=\sqrt{p_{s 1}[1] g_{s 1}^{s 2}[1]} X_{s 1}[1]+Z_{s 1}^{s 2}[1]
$$

In the second time slot, to trade for node $s 1$ 's relaying, SU node $s 2$ is willing to amplify $Y_{s 1}^{s 2}[1]$ and relay it to SU destination node $d 1$ over its otherwise wasted spectrum hole $\mathrm{CH} 1$. The received signal at $d 1$ is

$$
Y_{s 2}^{d 1}[2]=\sqrt{p_{s 2}[2] g_{s 2}^{d 1}[2]} X_{s 2}[2]+Z_{s 2}^{d 1}[2],
$$

where

$$
X_{s 2}[2]=Y_{s 1}^{s 2}[1] /\left|Y_{s 1}^{s 2}[1]\right| \text {. }
$$

is the transmitted signal from node $s 2$ to node $d 1$ which is normalized to have unit energy.

We assume the noises in different channels are independent and identically distributed (i.i.d.) with zero-mean and variance $\sigma^{2}$. Substituting (4) into (3), we can rewrite (3) as 


$$
Y_{s 2}^{d 1}[2]=\frac{\sqrt{p_{s 2}[2] g_{s 2}^{d 1}[2]}\left(\sqrt{p_{s 1}[1] g_{s 1}^{s 2}[1]} X_{s 1}[1]+Z_{s 1}^{s 2}[1]\right)}{\sqrt{p_{s 1}[1] g_{s 1}^{s 2}[1]+\sigma^{2}}}+Z_{s 2}^{d 1}[2] \text {. }
$$

Consequently, the received signal-to-noise ratio (SNR) at $\mathrm{SU}$ destination node $d 1$ helped by $s 2$ can be expressed as

$$
\eta_{1}=\frac{p_{s 1}[1] g_{s 2}^{s 2}[1] p_{s 2}[2] g_{s 2}^{d 1}[2]}{\sigma^{2}\left(p_{s 1}[1] g_{s 1}^{s 2}[1]+p_{s 2}[2] g_{s 2}^{d 1}[2]+\sigma^{2}\right)} .
$$

And the effective SNR of the AF cooperative channel of node $s i$ helped by node $s j$ is given by

$$
\Gamma_{1}=\eta_{1}+\gamma_{1}
$$

where $\gamma_{1}=p_{s 1}[1] g_{s 1}^{s 2}[1] / \sigma^{2}$ is the SNR that results from the direct transmission from node $s 1$ to node $d 1$ in the first time slot. Here, we use the channel capacity to represent the performance of each SU pair in our cooperative system. The resultant cooperative channel capacity of SU pair 1 can be expressed as

$$
C_{1}^{C}=\frac{1}{2} \log _{2}\left(1+\Gamma_{1}\right) .
$$

The coefficient $1 / 2$ in (8) is due to the fact that the transmission time of a SU pair will occupy two time slots. If SU node $s 1$ refuses to relay SU node $s 2$ 's signal, $s 2$ has no reason to act as a relay for $s 1$. SU pair $s 1$ will work independently, so the noncooperative channel capacity of SU pair 1 is

$$
C_{1}^{N}=\frac{1}{2} \log _{2}\left(1+\gamma_{1}\right) \text {. }
$$

In practical networks, users' transmission powers are limited and each node has several discrete power levels for transmission. Assume both SU source nodes have $L$ transmit power levels, $P^{1}, P^{2}$, $\ldots, P^{L}, P^{1}<P^{2}<\ldots<P^{L}$. Meanwhile, users are also energy limited by the battery capacity. To keep within the energy limit, we suppose there is a frame energy constraint for node $s 1$, i.e.

$$
\frac{p_{s 1}[1] T}{2}+\frac{p_{s 1}[2] T}{2} \leq E
$$

where $E$ is the frame energy constraint for both SU source nodes. Furthermore, only limited interference with primary systems is allowed in CR systems. Thus, to prevent SUs from causing severe interference to PUs, the transmission powers of $s 1$ and $s 2$ must also satisfy

$$
\begin{aligned}
& p_{s 1}[1] g_{s 1}^{1}[1] \leq I_{1}, \\
& p_{s 2}[1] g_{s 2}^{2}[1] \leq I_{2}, \\
& p_{s 1}[2] g_{s 1}^{2}[2] \leq I_{2}, \\
& p_{s 2}[2] g_{s 2}^{1}[2] \leq I_{1},
\end{aligned}
$$

where $I_{1}, I_{2}$ are the acceptable interference powers of primary users over $\mathrm{CH} 1$ and $\mathrm{CH} 2$, respectively, which can be determined according to their QoS requirements.

\section{Problem Formulation and Game Model}

Since mobile users in practical networks are energy limited, we assume both SU pairs aim at maximizing their cooperative channel capacity by consuming the same amount of energy. Based on the former analysis, we define the cooperation strategy of SU pair $i$ as the transmission power of $s i, p_{s i}[1]$, and the cooperative transmission power, $p_{s i}[2]$. From (6), (7) and (8), we notice that the cooperative channel capacity of SU pair $1, C_{1}$ is determined by not only its own strategy $p_{s 1}[1]$ in the first time slot, but also the strategy $p_{s 2}$ [2] of SU pair 2 in the second time slot. Due to the symmetry of our model, the capacity of SU pair 2 is also determined by the strategy of SU pair 1 and itself. The cooperative strategy reflects that the rational decision made by one SU pair will definitely affect the other SU pair's choice.

Since both SU pairs aim at maximizing their capacity, they have to share their power resource to seek cooperative relaying, which means both SU pairs would like to negotiate with each other and reach an agreement on how to allocate the power resource. We formulate this power allocation 
problem as a cooperative game, i.e., the power allocation game (PAG). Through this game, a payoff allocation which is said to be Pareto optimal can be achieved and the two specified network objectives efficiency and fairness can be implemented.

Formally, let $K=\{1,2\}$ be the set of players (SU pairs) in the PAG. We define the set of feasible payoffs for SU pair $i$ as $Q_{i}=\left\{R_{i} \mid R_{i}=U_{i}-U_{i}^{\text {min }}\right\}$, where $U_{i}^{\text {min }}=C_{i}^{N}$ represents the non-cooperative payoff for SU pair $i$, and $U_{i}=C_{i}^{C}$ represents the cooperative payoff for SU pair $i$.

$$
R_{i}=\frac{1}{2}\left[\log _{2}\left(1+\Gamma_{i}\right)-\log _{2}\left(1+\gamma_{i}\right)\right] \text {. }
$$

By the definition of $Q_{i}, s i$ will quit cooperation when its payoff is less than $U_{i}^{\min }$. That ensures a SU pair would participate in cooperative relaying only if the capacity achieved through cooperation will be higher than that achieved through noncooperation by utilizing the same amount of energy. Denote the set of feasible payoffs that both cooperative SU pairs can get as $\boldsymbol{Q}=\left\{\boldsymbol{R}=\left(R_{1}, R_{2}\right) \mid R_{1} \in Q_{1}, R_{2} \in Q_{2}\right\}$. The game $\{K, Q\}$ is a bargaining problem if and only if $Q$ is a convex subset of $Q^{K}[11]$, which means for any $0 \leq \theta \leq 1$, if $\boldsymbol{R}^{\alpha}=\left(R_{1}^{\alpha}, R_{2}^{\alpha}\right) \in Q$ and $\boldsymbol{R}^{\beta}=\left(R_{1}^{\beta}, R_{2}^{\beta}\right) \in Q$, then $\theta \boldsymbol{R}^{\alpha}+(1-\theta) \boldsymbol{R}^{\beta} \in \boldsymbol{Q}$.

Proof: Substituting (15) into $\theta R_{1}^{\alpha}+(1-\theta) R_{1}^{\beta}$, we can get

$$
\theta R_{1}^{\alpha}+(1-\theta) R_{1}^{\beta}=\frac{1}{2}\left[\theta \log _{2}\left(1+\Gamma_{1}^{\alpha}\right)-(1-\theta) \log _{2}\left(1+\Gamma_{1}^{\beta}\right)\right]-\frac{1}{2}\left[\theta \log _{2}\left(1+\gamma_{1}^{\alpha}\right)-(1-\theta) \log _{2}\left(1+\gamma_{1}^{\beta}\right)\right] .
$$

From (7), we know that $\Gamma_{1}$ is a function of $p_{s 1}[1]$ and $p_{s 2}[2]$, and $\log _{2}\left(1+\Gamma_{1}\right)$ is a monotonically increasing function of $\Gamma_{1}$. There must exist at least one point $\left(p_{s 1}^{\max }[1], p_{s 2}^{\max }[2]\right), P^{1} \leq p_{s 1}^{\max }[1], p_{s 2}^{\max }[2] \leq P^{L}$, at which $\Gamma_{1}\left(p_{s 1}[1], p_{s 2}[2]\right)$ reaches its maximum $\Gamma_{1}^{\max }\left(p_{s 1}^{\max }[1], p_{s 2}^{\max }[2]\right)$ and, as a result, $\log _{2}\left(1+\Gamma_{1}\right)$ reaches its maximum $\log _{2}\left(1+\Gamma_{1}^{\max }\right)$. Also, there must exist at least one point ( $\left.p_{s 1}^{\min }[1], p_{s 2}^{\min }[2]\right)$, $P^{1} \leq p_{s 1}^{\min }[1], p_{s 2}^{\min }[2] \leq P^{L}$, at which $\Gamma_{1}\left(p_{s 1}[1], p_{s 2}[2]\right)$ reaches its minimum $\Gamma_{1}^{\min }\left(p_{s 1}^{\min }[1], p_{s 2}^{\min }[2]\right)$ and, as a result, $\log _{2}\left(1+\Gamma_{1}\right)$ reaches its minimum $\log _{2}\left(1+\Gamma_{1}^{\min }\right)$. Then we get

$$
\log _{2}\left(1+\Gamma_{1}^{\min }\right) \leq \log _{2}\left(1+\Gamma_{1}\right) \leq \log _{2}\left(1+\Gamma_{1}^{\max }\right) .
$$

In (17), because $P^{1} \leq p_{s 1}^{\alpha}[1], p_{s 2}^{\alpha}[2] \leq P^{L}, \Gamma_{1}^{\alpha}=\Gamma_{1}^{\alpha}\left(p_{s 1}^{\alpha}[1], p_{s 2}^{\alpha}[2]\right), P^{1} \leq p_{s 1}^{\beta}[1], p_{s 2}^{\beta}[2] \leq P^{L}$ and $\Gamma_{1}^{\beta}=\Gamma_{1}^{\beta}\left(p_{s 1}^{\beta}[1], p_{s 2}^{\beta}[2]\right)$, we can get $\Gamma_{1}^{\min } \leq \Gamma_{1}^{\alpha} \leq \Gamma_{1}^{\max }$ and $\Gamma_{1}^{\min } \leq \Gamma_{1}^{\beta} \leq \Gamma_{1}^{\max }$. And it is easy to derive that

$$
\log _{2}\left(1+\Gamma_{1}^{\min }\right) \leq \log _{2}\left(1+\Gamma_{1}^{\alpha}\right) \leq \log _{2}\left(1+\Gamma_{1}^{\max }\right) \text { and } \log _{2}\left(1+\Gamma_{1}^{\min }\right) \leq \log _{2}\left(1+\Gamma_{1}^{\beta}\right) \leq \log _{2}\left(1+\Gamma_{1}^{\max }\right) \text {. }
$$

For any $0 \leq \theta \leq 1$, we then have

$$
\log _{2}\left(1+\Gamma_{1}^{\min }\right) \leq \theta \log _{2}\left(1+\Gamma_{1}^{\alpha}\right)+(1-\theta) \log _{2}\left(1+\Gamma_{1}^{\beta}\right) \leq \log _{2}\left(1+\Gamma_{1}^{\max }\right) .
$$

From (17) and (18), we can conclude that the term $\theta \log _{2}\left(1+\Gamma_{1}^{\alpha}\right)+(1-\theta) \log _{2}\left(1+\Gamma_{1}^{\beta}\right)$ in (16) has the same value space as the term $\log _{2}\left(1+\Gamma_{1}\right)$.

For the term $\log _{2}\left(1+\gamma_{1}\right)$ in (16), because $P^{1}<p_{s 1}[1]<P^{L}$, we can get the value space of $\gamma_{1}$ is $\left[P^{1} g_{s 1}^{s 2}[1] / \sigma^{2}, P^{L} g_{s 1}^{s 2}[1] / \sigma^{2}\right]$. Since $\log _{2}\left(1+\gamma_{1}\right)$ is also a monotonically increasing function of $\gamma_{1}$. The value space of $\log _{2}\left(1+\gamma_{1}\right)$ is

$$
\left[\log _{2}\left(1+P^{1} g_{s 1}^{s 2}[1] / \sigma^{2}\right), \log _{2}\left(1+P^{L} g_{s 1}^{s 2}[1] / \sigma^{2}\right)\right] \text {. }
$$

For the term $\theta \log _{2}\left(1+\gamma_{1}^{\alpha}\right)+(1-\theta) \log _{2}\left(1+\gamma_{1}^{\beta}\right)$ in (16), because $P^{1} \leq p_{s 1}^{\alpha}[1] \leq P^{L}$ and $P^{1} \leq p_{s 1}^{\beta}[1] \leq P^{L}$ both the value space of $\gamma_{1}^{\alpha}=p_{s 1}^{\alpha}[1] g_{s 1}^{s 2}[1] / \sigma^{2}$ and $\gamma_{1}^{\beta}=p_{s 1}^{\beta}[1] g_{s 1}^{s 2}[1] / \sigma^{2}$ are $\left[P^{1} g_{s 1}^{s 2}[1] / \sigma^{2}, P^{L} g_{s 1}^{s 2}[1] / \sigma^{2}\right]$. We can prove that, for any $0 \leq \theta \leq 1$, the term $\theta \log _{2}\left(1+\gamma_{1}^{\alpha}\right)+(1-\theta) \log _{2}\left(1+\gamma_{1}^{\beta}\right)$ in $(16)$ and the term $\log _{2}\left(1+\gamma_{1}\right)$ in (9) have the same value space $\left[\log _{2}\left(1+P^{1} g_{s 1}^{s 2}[1] / \sigma^{2}\right), \log _{2}\left(1+P^{L} g_{s 1}^{s 2}[1] / \sigma^{2}\right)\right]$. And then we can prove that $\theta R_{1}^{\alpha}+(1-\theta) R_{1}^{\beta} \in R_{1}$.

By the same method, we can also prove that $\theta R_{2}^{\alpha}+(1-\theta) R_{2}^{\beta} \in R_{2}$. Therefore, $\theta \boldsymbol{R}^{\alpha}+(1-\theta) \boldsymbol{R}^{\beta} \in \boldsymbol{Q}$, and then $\boldsymbol{R}=\left(R_{1}, R_{2}\right)$ is a convex set.

We have proved that the proposed PAG is indeed a two-person bargaining problem. The unique solution of the PAG can be solved by using the Nash bargaining solution (NBS) method [12]. Thus, the 
NBS strategy profile of both cooperative SU pairs can be found by solving the following maximization problem

$$
\left(p_{s 1}^{*}[1], p_{s 1}^{*}[2], p_{s 2}^{*}[1], p_{s 2}^{*}[2]\right)=\arg \max R_{1} R_{2},
$$

subject to

$P^{1} \leq p_{s 1}[1], p_{s 1}[2], p_{s 1}[1], p_{s 2}[2] \leq P^{L} \quad, \quad p_{s 1}[1] g_{s 1}^{1}[1] \leq I_{1} \quad, \quad p_{s 2}[1] g_{s 2}^{2}[1] \leq I_{2} \quad, \quad p_{s 1}[2] g_{s 1}^{2}[2] \leq I_{2} \quad, \quad p_{s 2}[2] g_{s 2}^{1}[2] \leq I_{1} \quad ，$ $\frac{p_{s 1}[1] T}{2}+\frac{p_{s 1}[2] T}{2} \leq E$

Although a closed-form solution for problem (20) exists, it is omitted here because of the limitation of the space. Note that (20) is a combinatorial problem in nature which involves four discrete variables. A numerical algorithm can be used to search the NBS of the PAG with a computation complexity of $\mathrm{O}(\mathrm{N} 2)$. The knowledge required by a node to perform the PAG and to use the maximal ratio combining (MRC) is the channel state information (CSI), which includes that for source-destination, source-relay, and relay-destination channels of its own and the partner. This information can be acquired by the dedicated feedback channels. Here, we assume the channels are slow fading and remain constant over any time slot. Therefore all channel gains are fixed during the time of interest.

\section{Simulation Results}

In this section, we present simulation results of the proposed games. To evaluate the fairness and efficiency performances of the game, we compare the NBS power allocations with the maximal-rate optimization. The objective of the maximal-rate optimization is to maximize the overall system rate. This requires that all user nodes are fully cooperative. The maximal-rate cooperative resource allocation can be achieved by solving the following optimization problem $\arg \min \left(C_{1}+C_{2}\right)$.

The path gain is set to $0.097 / d^{4}$, where $d$ is the distance between the transmitter and the receiver (in meters). The noise level is $\sigma^{2}=1 \times 10^{-14} \mathrm{~W}$. The minimum and maximum power levels for a transmission node is $P^{1}=1 \mathrm{~mW}$ and $P^{L}=10 \mathrm{~mW}$, respectively. A simulated system is illustrated in Fig.2. We locate the Source 1 at $(0,0)$, Destination 1 at $(1500,0)$, and Destination 2 at $(-1000,0)$, and fix the $x$ coordinate of Source 2 at $\mathrm{x}=500$ while increase its y coordinate from -600 to 600 .

Let $\mathrm{Y}_{2}$ denote the y coordinate of Source 2. It can be find in fig.2 that PAG only has a little system throughput loss than Max Rate, and it can be see clearly that the both users in PAG have more balanced capacity than in Max Rate.

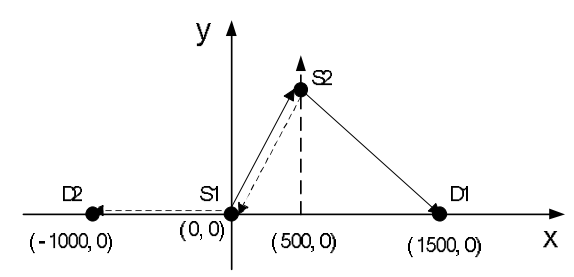

Fig. 1 CR cooperative transmission system model 


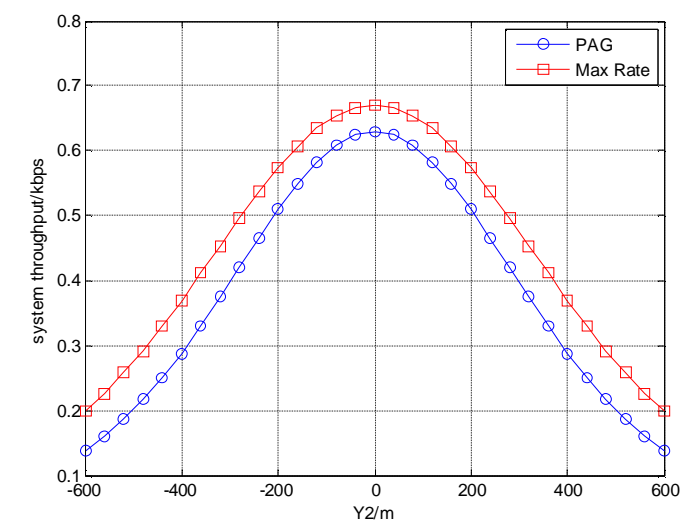

Fig. 2 system throughput in different adaptive power allocation schemes

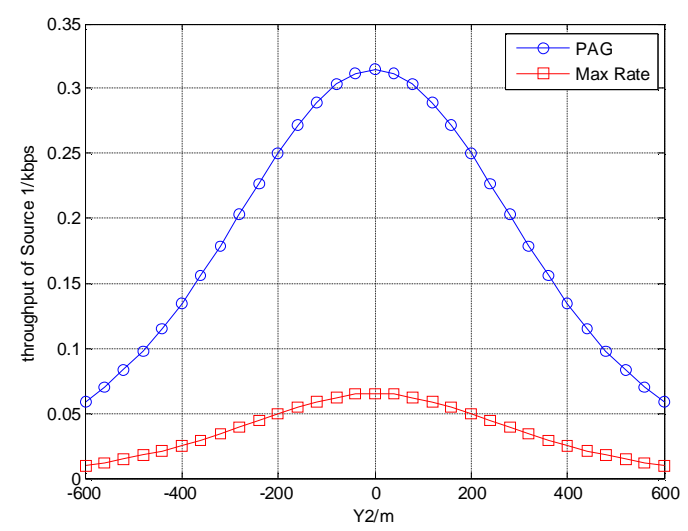

Fig. 3 Throughput of user 1 in different adaptive resource allocation schemes

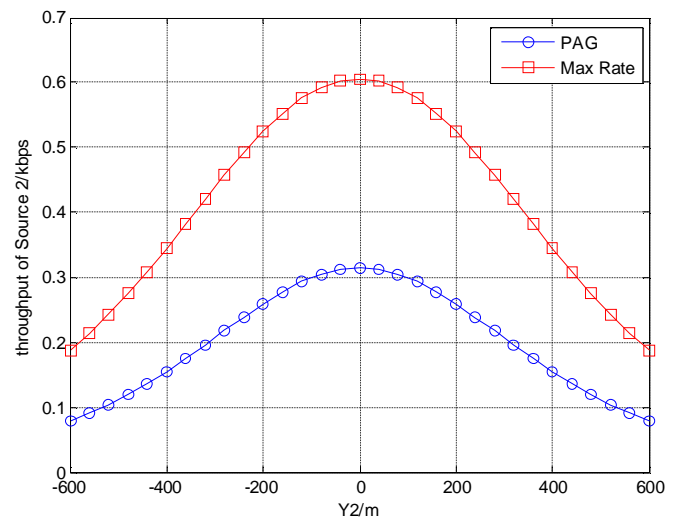

Fig. 4 Throughput of user 2 in different adaptive resource allocation schemes

\section{Conclusions}

In this paper, we have analyzed the cooperation behavior of selfish user nodes in cooperative CR communication networks. We formulate the energy-efficient resource allocation problem among two cooperating node pairs as cooperative game, and use the Nash bargaining solution (NBS) method to obtain the solution of the game. By comparing with the maximal-rate optimization scheme, simulation results show the NBS resource allocation is efficient in that the performance loss of the NBS to that of the maximal-rate optimization is small while the maximal-rate scheme is extremely unfair. The simulation results also show that the NBS resource allocation is fair in that both nodes could experience better performance than they work independently. 


\section{References}

[1] J. Mitola et al., “ Cognitive Radio: Making Software Radios More Personal,” IEEE Pers. Commun., Aug. 1999, pp. 13- 18.

[2] Q. Zhang, J. Jia, and J. Zhang, “ Cooperative relay to improve diversity in cognitive radio networks," IEEE Commun. Mag., vol. 47, no. 2, pp. 111- 117, Feb. 2009.

[3] G. Zhao, C. Yang, G. Y. Li, D. Li, and A. C. K. Soong, “ Power and channel allocation for cooperative relay in cognitive radio networks," IEEE J. Sel. Topics Signal Process. , vol. 5, no. 1, pp. 151- 159, Feb. 2011.

[4] K. Letaief and W. Zhang, " Cooperative communications for cognitive radio networks," Proc. IEEE, vol. 97, pp. 878- 893, May 2009.

[5] J. Mietzner, L. Lampe, and R. Schober, “ Distributed transmit power allocation for multihop cognitive-radio systems," IEEE Trans. Wireless Commun., vol. 8, no. 10, pp. 5187- 5201, Oct. 2009.

[6] L. Li, X. Zhou, H. Xu, and G. Y. Li, “ Simplified relay selection and power allocation in cooperative cognitive radio systems," IEEE Trans. Wireless Commun., vol. 10, no. 1, pp. 33- 36, Jan. 2011.

[7] J. Huang, et al., " Auction-based distributed resource allocation for cooperation transmission in wireless networks," in Proc. IEEE GLOBECOM' 07, 2007, pp. 4807-4812.

[8] G. Zhang, et al., “ Competitive resource sharing based on game theory in cooperative relay networks," ETRI Journal, vol. 31, no. 1, pp. 89-91,Feb. 2009.

[9] G. Zhang, L. Cong, K. Yang, et al, “ Competitive Resource Sharing Based on Game Theory in Cooperative Relay Networks," ETRI Journal, 2009, 31(1), pp. 89-91.

[10]L. Cong, G. Zhang, and K. Yang, et al, “ A Stackelberg game for resource allocation in multiuser cooperative transmission networks," Wirel. Commun. Mob. Comput. 2010, 1-21.

[11]B. Wang, et al., "Distributed relay selection and power control for multiuser cooperative communication networks using buyer/seller game," in Proc. IEEE INFOCOM '07, 2007, pp. 544-552.

[12]H. Yaiche, R. R. Mazumdar, and C. Rosenberg, “ A game theoretic framework for bandwidth allocation and pricing in broadband networks," IEEE/ACM Trans. Networking, vol. 8, no. 5, pp. 667-678, 2000. 